\title{
Active loaded plasmonic antennas at terahertz frequencies: Optical control of their capacitive-inductive coupling
}

\author{
G. Georgiou, ${ }^{1, *}$ C. Tserkezis, ${ }^{2}$ M. C. Schaafsma,${ }^{1}$ J. Aizpurua,${ }^{2}$ and J. Gómez Rivas ${ }^{1,3, \dagger}$ \\ ${ }^{1}$ Center for Nanophotonics, FOM Institute AMOLF, Science Park 104, 1098 XG, Amsterdam, The Netherlands \\ ${ }^{2}$ Donostia International Physics Center (DIPC) and Centro de Física de Materiales (CFM) CSIC-UPV/EHU, \\ Paseo Manuel de Lardizabal 4, Donostia-San Sebastián 20018, Spain \\ ${ }^{3}$ COBRA Research Institute, Eindhoven University of Technology, P.O. Box 513, 5600 MB, Eindhoven, The Netherlands
}

(Received 6 January 2015; revised manuscript received 19 February 2015; published 31 March 2015)

\begin{abstract}
We demonstrate the photogeneration of loaded dipole plasmonic antennas resonating at $\mathrm{THz}$ frequencies. This is achieved by the patterned optical illumination of a semiconductor surface using a spatial light modulator. Our experimental results indicate the existence of capacitive and inductive coupling of localized surface plasmon polaritons. By varying the load in the antenna gap we are able to switch between both coupling regimes. Furthermore, we determine experimentally the effective impedance of the antenna load and verify that this load can be effectively expressed as a $L C$ resonance formed by a $\mathrm{THz}$ inductor and capacitor connected in a parallel circuit configuration. These findings are theoretically supported by full electrodynamic calculations and by simple concepts of lumped circuit theory. Our results open new possibilities for the design of active THz circuits for optoelectronic devices.
\end{abstract}

DOI: 10.1103/PhysRevB.91.125443

PACS number(s): 73.20.Mf, 78.67.Pt, 78.20.-e, 07.57.Pt

\section{INTRODUCTION}

Optical antennas are crucial components in the design of future optoelectronic devices. Their main function is to convert free-space electromagnetic radiation into localized energy and vice versa [1]. Over the past decade plasmonic antennas have gained an exceptional popularity mainly because of their ability to confine visible and infrared radiation into lengths much smaller than the free-space wavelength [2]. Their prospective use in nanoscale electronic devices and detectors [3-5] as well as in systems that arbitrarily control wavefronts [6] has stimulated many research groups to thoroughly investigate their optical far-field properties and near-field interactions with neighboring elements. The configurations that have been proposed, inspired by their radio-frequency analogs, include among others, dipole [7], Yagi-Uda [8,9], and bowtie [10-12] antennas whose purpose is to control directivity and bandwidth of visible and infrared waves. Furthermore, new antenna designs that exhibit more exotic properties have been proposed recently. These designs combine multiple resonating elements to achieve asymmetric Fano-like resonances [13-18] and electromagnetically induced transparency $[19,20]$ as well as metasurfaces that introduce gradual phase retardation along the propagation direction $[6,21,22]$.

In addition to these studies, a great emphasis has been given to the investigation of actively controlling the optical properties of plasmonic antennas. For this purpose dielectric and metallic loading of antenna elements has been considered and lumped circuit theory has been used to describe their resonant properties [23-25]. Near-field optical microscopy has been used to study the amplitude and phase of the fields in loaded nanoantennas verifying the validity of the lumped circuit theory at the nanoscale [26]. A similar design has been used recently to investigate the scattering and nonlinear optical

\footnotetext{
*georgiou@amolf.nl

†rivas@amolf.nl
}

properties of such loaded antennas [27,28]. These works have laid a solid ground for actively controlling the properties of metallic antennas.

Semiconducting materials could be the ultimate candidate for actively controllable antennas, owing to their versatile nature. Their optical properties can be easily adjusted by simply controlling the density of free charge carriers, which leads also into a tunability of their plasmonic behavior from the infrared to the far-infrared $(\mathrm{THz})$ regime [29,30]. Several studies have exploited the flexible nature of semiconductors in combination with metals to achieve tunability of resonances in plasmonic and metamaterial structures [31-34]. Besides these studies, an all-optical control of plasmonic antennas made completely out of semiconductors has been recently demonstrated using a spatial light modulator (SLM) [35].

In this paper we present an experimental and theoretical study on photogenerated coupled plasmonic antennas, made out of semiconductors and functioning at $\mathrm{THz}$ frequencies. Furthermore, we explore the photoexcitation of loads at the gaps of these antennas that act as capacitors or inductors, and discuss the optical response of the antennas in terms of a simple electrical circuit model. In contrast to previous studies [26,27], using the information of the complex electric field provided by $\mathrm{THz}$ time domain spectroscopy we are able to determine experimentally an effective impedance of the equivalent resonant $L C$ circuit. Finally, we observe the existence of a capacitive and an inductive behavior of localized surface plasmon polaritons formed at different frequencies.

\section{PHOTOEXCITATION OF ACTIVE LOADED ANTENNAS}

Our experiments have been performed using a timeresolved $\mathrm{THz}$ time domain spectrometer (TDS). For this pump-probe technique a commercial amplified laser system has been utilised (Legend Elite $\mathrm{HE}+$ seeded with the Mantis Ti:Sapphire laser). The laser delivers $100 \mathrm{fs}$ amplified pulses 

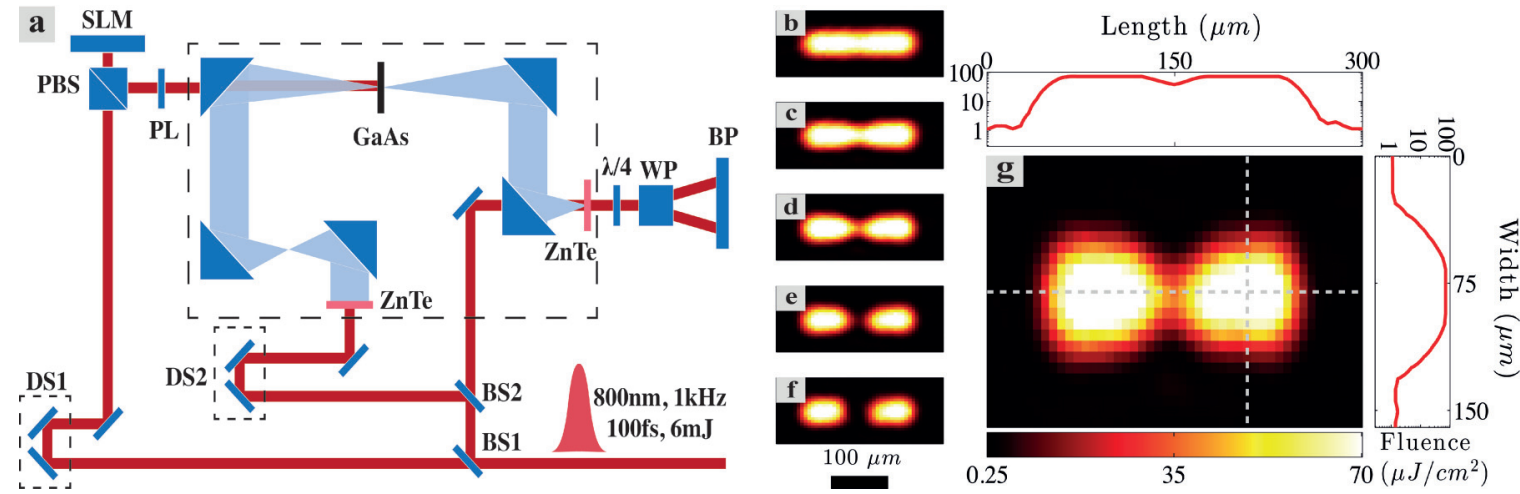

FIG. 1. (Color online) Photogenerated dimer THz antennas. (a) Schematic of a time-resolved THz spectrometer (DS: delay stage; BS: beam splitter; PBS: polarizing beam splitter; PL: projection lens; WP: Wollaston prism; BP: balanced photodiode). (b) The two components of the coupled antennas are merged together forming a single linear antenna. (f) The coupled antennas are separated by a dielectric gap with length $L_{\mathrm{g}}=53 \pm 3 \mu \mathrm{m}$. (c)-(e) Intermediate cases where the two constituent components of the dimer antenna are bridged with a metalliclike connector. (g) shows a detailed analysis of (d). The color scale and the vertical scale of the horizontal and vertical cuts illustrates the pump fluence.

with wavelength $\lambda=800 \mathrm{~nm}$ and pulse energy $6 \mathrm{~mJ}$ at $1 \mathrm{KHz}$ repetition rate. These pulses are used for $\mathrm{THz}$ generation and detection as well as for band-to-band carrier excitation in the semiconducting sample. For the generation and detection of $\mathrm{THz}$ pulses we use $20 \%$ of the intensity. Out of this intensity $95 \%$ is used for $\mathrm{THz}$ generation through difference frequency mixing in a $0.5 \mathrm{~mm} \mathrm{ZnTe}$ crystal, while the rest is used for electrooptical detection in a $1 \mathrm{~mm} \mathrm{ZnTe}$ crystal as illustrated in Fig. 1(a). By controlling the time delay between the beam that generates the $\mathrm{THz}$ radiation and the one used for the detection we can reconstruct the $\mathrm{THz}$ electric field transients. The remaining $80 \%$ of the initial intensity is used for photoexciting free carriers in the semiconductor. This beam, which is spatially shaped by a SLM and projected on the sample, excites free electrons at the illuminated areas of a semiconductor surface in the form of coupled dimer antennas (Fig. 1(b)-1(g) and Fig. S-1 [36]). The sample used for the experiments is a $1 \mu \mathrm{m}$ thick single crystalline GaAs bonded on to a $1 \mathrm{~mm}$ thick amorphous quartz substrate [35].

The pump-probe time delay $\Delta \tau_{\mathrm{p}-\mathrm{p}}$ in our experiment is defined as the temporal separation between the optical pump pulse, which excites the free charge carriers and the $\mathrm{THz}$ probe pulse. With this definition a $\Delta \tau_{\mathrm{p}-\mathrm{p}}<0$ indicates that the $\mathrm{THz}$ pulse propagates through the semiconductor before the excitation of free carriers. On the other hand $\Delta \tau_{\mathrm{p}-\mathrm{p}}>0$ implies that the $\mathrm{THz}$ pulse propagates through the semiconductor after the free carriers have been excited by the pump pulse. A $\Delta \tau_{\mathrm{p}-\mathrm{p}}=10 \mathrm{ps}$ was chosen for our experiments. This time delay is sufficiently short, such that the spatial diffusion of the free carriers does not modify the dimensions of the photoexcited structures. It is also essential to mention that at this time scale we did not observe any ballistic electrons that could potentially modify the dimensions of the structures. The dynamics of the photoexcited carriers display an almost single-exponential behavior that leads to an effective carrier recombination time, which includes surface and bulk recombination, of around $\tau_{\mathrm{r}} \sim 300 \mathrm{ps}$ (see Fig. S-2 in Supplemental Material). Moreover, the number of photoexcited carriers remains unchanged during the propagation of the $\mathrm{THz}$ pulse through the sample since its temporal envelope $\tau_{\mathrm{THz}}$ is much shorter than the recombination time $\tau_{\mathrm{r}}$ of the free charge carriers in $\operatorname{GaAs}\left(\tau_{\mathrm{THz}} \sim 1 \mathrm{ps} \ll \tau_{\mathrm{r}}\right)$.

Figures 1(b)-1(f) show images of the photoexcited structures taken by placing a CCD camera at the sample position. As can be appreciated in this figure the total length and width of the antennas is kept constant and only the intensity in the gap is varied. Figure $1(\mathrm{~g})$ represents a more detailed analysis of Fig. 1(d). To have an estimate of the photoinduced antenna dimensions, we define an effective length and width by taking the size at which the pump intensity drops at half of its maximum (FWHM). This gives an effective length of $L=195 \pm 3 \mu \mathrm{m}$ and width of $W=56.5 \pm 3 \mu \mathrm{m}$, where the uncertainties in the estimated values are associated with half of the pixel size of the CCD camera. The single linear antenna [Fig. 1(b)] is being split into two coupled antennas that are connected with a bridge that has constant effective length of $L_{\mathrm{g}}=53 \pm 3 \mu \mathrm{m}$ and varying width $W_{\mathrm{b}}$ from $0-56.5 \mu \mathrm{m}$. Determining the bridge dimensions as well as the carrier concentration is critical. The bridge width is defined by summing the number of pixels in the gap area that have an intensity larger than the intensity at half the maximum (FWHM). Taking into consideration the pixel dimensions $\left(6.45^{2} \mu \mathrm{m}^{2}\right)$ of the camera, this calculation will result into an effective area that has intensity higher than half the maximum. Subsequently, we define the effective bridge width, $W_{\mathrm{b}}$, by dividing the calculated area by the gap length $L_{\mathrm{g}}$. The values of $W_{\mathrm{b}}$ are reported in the insets of Fig. 2 for each case. The photogenerated loaded antennas are positioned in a random distribution (see Fig. S-1 in Supplemental Material [36]) with average density of $\sim 9.5$ antennas $/ \mathrm{mm}^{2}$. The random distribution ensures that features associated to diffractive orders in the far-field spectrum are suppressed.

As it can be seen by the detailed analysis on one of the intermediate antennas [Fig. 1(g)], the maximum pump fluence is $70 \mu \mathrm{J} / \mathrm{cm}^{2}$. To understand the behavior of our sample at these high pump fluences we have performed a pump-probe analysis. In this analysis we have measured the $\mathrm{THz}$ transmission through a homogeneously pumped GaAs layer. From this measurement we have determined the conductivity of the sample, which has a Drude-like behavior 


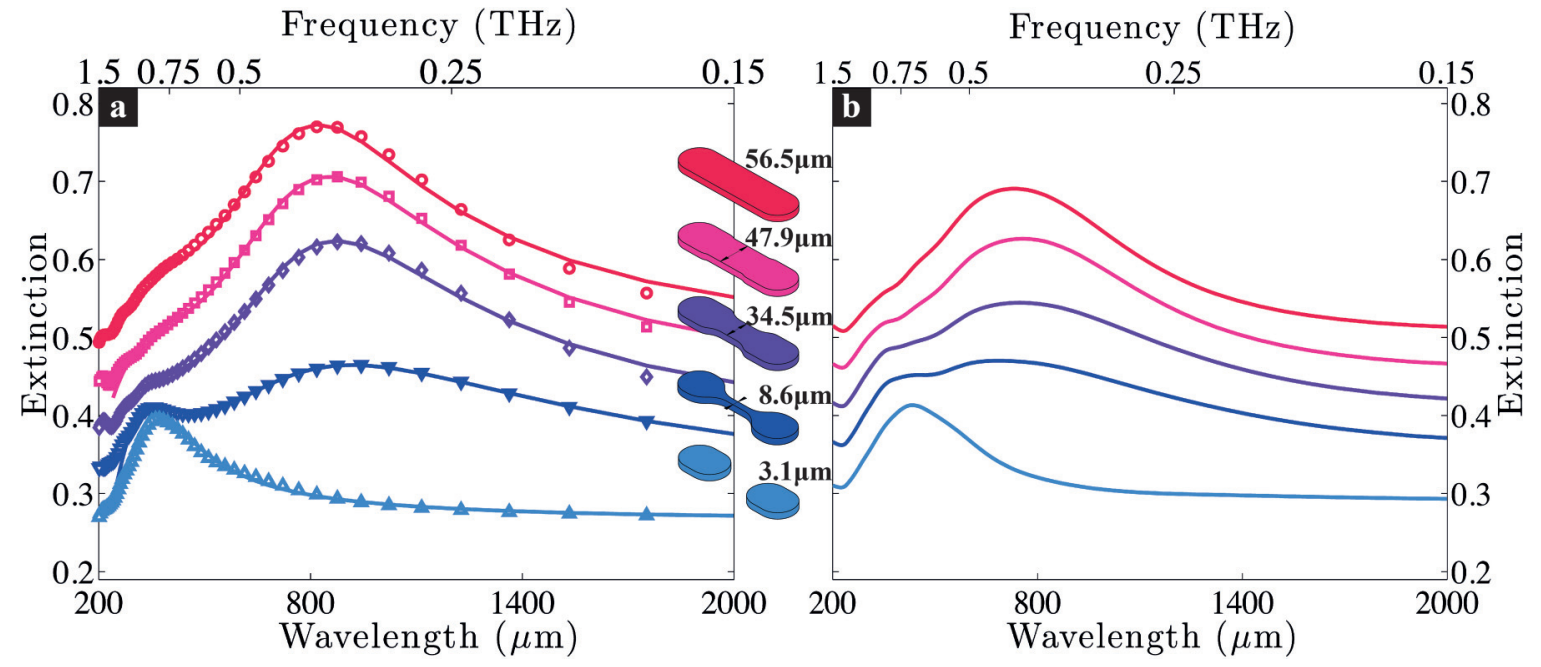

FIG. 2. (Color online) (a) Far-field extinction measurements of randomly positioned loaded antennas with different bridge widths, as shown in the insets. The symbols are the experimental data and the solid curves represent bi-Lorentzian fits to the data. (b) Numerical FDTD calculations of far field extinction. The curves are displaced for clarity by a vertical shift of $0.2,0.15,0.1,0.05$, and 0 from red to blue respectively.

with carrier density $N=2 \times 10^{18} \mathrm{~cm}^{-3}$ and carrier mobility of $\mu=0.15 \mathrm{~m}^{2} \mathrm{~V}^{-1} \mathrm{~s}^{-1}$. It is worthwhile to note that the finite contrast of the image projected by the SLM leads to a finite pump fluence in the regions defined as dark by the SLM. This fluence is lower than $<0.25 \mu \mathrm{J} / \mathrm{cm}^{2}$. A detailed analysis on a transmission measurement from a fully dark image, as defined by the SLM, showed that the conductivity of the sample follows a Drude-like behavior with carrier density $N=3 \times 10^{15} \mathrm{~cm}^{-3}$ and mobility $\mu=0.7 \mathrm{~m}^{2} \mathrm{~V}^{-1} \mathrm{~s}^{-1}$.

\section{RESULTS}

To determine the $\mathrm{THz}$ response of the dimer antennas, we excite them at normal incidence and we measure the $\mathrm{THz}$ transmission spectrum in the forward direction. Figure 2(a) illustrates the main experimental results of this paper. This plot shows the far-field extinction spectra, defined as one minus the normalized transmittance, as a function of wavelength (bottom axis) and frequency (top axis). The extinction is equal to the sum of scattering and absorption. The symbols are the experimental data points while the solid lines are bi-Lorentzian fits to the data. The main feature of this figure is a resonance at long wavelengths that red shifts from $850 \mu \mathrm{m}$ to $1034 \mu \mathrm{m}$ as we decrease the width of the bridge. This resonance corresponds to the fundamental resonant mode of the plasmonic antennas $(\lambda / 2$ resonance), defined by an effective length that characterizes the entire loaded antenna as described above. Moreover, as the bridge width decreases (i.e., bridge width of $8.6 \mu \mathrm{m})$ a second resonance appears at shorter wavelengths $(\lambda=327 \mu \mathrm{m}$, dark blue curve). This resonance is slightly blue shifted with respect to the $\lambda / 2$ resonance of the individual arms of the dimer antenna at $\lambda=380 \mu \mathrm{m}$ (see light blue curve, which corresponds to $W_{\mathrm{b}}=3.1 \mu \mathrm{m}$ ). The transition from the $\lambda / 2$ resonance of the entire plasmonic antenna to the $\lambda / 2$ resonance of the dimer antenna is controlled by the width of the gap linking the two components. As it is elucidated later in the manuscript, this behavior can be explained by a simple lumped circuit model. Figure 2(b) represents full three-dimensional (3D) electrodynamic calculations of the far-field extinction using the finite difference in the time domain (FDTD Lumerical) method. The design of the structures used for the simulations incorporates a graded carrier concentration towards the edges of the antenna to mimic as close as possible the conditions of the experiment [35]. The simulations qualitative reproduce the main farfield resonant features of the experimental observations. The small quantitative discrepancies between measurements and simulations can be attributed to the difficulty in defining from the CCD images the exact antenna geometry and dimensions for the simulations.

Although the spectra from both the experiment and simulations provide qualitative information about the different modes of this system, it is important to investigate more in detail the interaction of the antenna with its load. Therefore, with use of the FDTD method we have numerically calculated the electric field enhancement $\left|\mathbf{E}(\mathbf{r}, \omega) / \mathbf{E}_{0}(\mathbf{r}, \omega)\right|$ and the surface charge $\sigma(\mathbf{r}, \omega)$ distribution of one of the antennas $\left(W_{\mathrm{b}}=8.6 \mu \mathrm{m}\right)$, at the short- and long- wavelength resonances. We should note that the obtained surface charge distributions have been smoothed, in order to remove the artificial (much lower) surface charges induced by the sequential shells of graded carrier concentrations. In Fig. 3(a) we show the electric field enhancement at $\lambda=327 \mu \mathrm{m}$, calculated at the middle of the antenna's height, i.e. $0.5 \mu \mathrm{m}$. The corresponding surface charge distribution $\sigma(\mathbf{r}, \omega)$ is displayed in Fig. 3(b), where a dipole-dipole distribution typical of a bonding mode can be observed [37]. This mode, which is slightly blue shifted with respect to the corresponding mode in the absence of a conducting bridge (see Fig. 2), indicates a capacitive load.

On the other hand, the presence of the photoinduced bridge allows for charge transfer along the entire antenna, which manifests itself by the appearance of a charge transfer mode at longer wavelengths [37]. In the particular case we are examining here, due to the small width of the bridge, this 

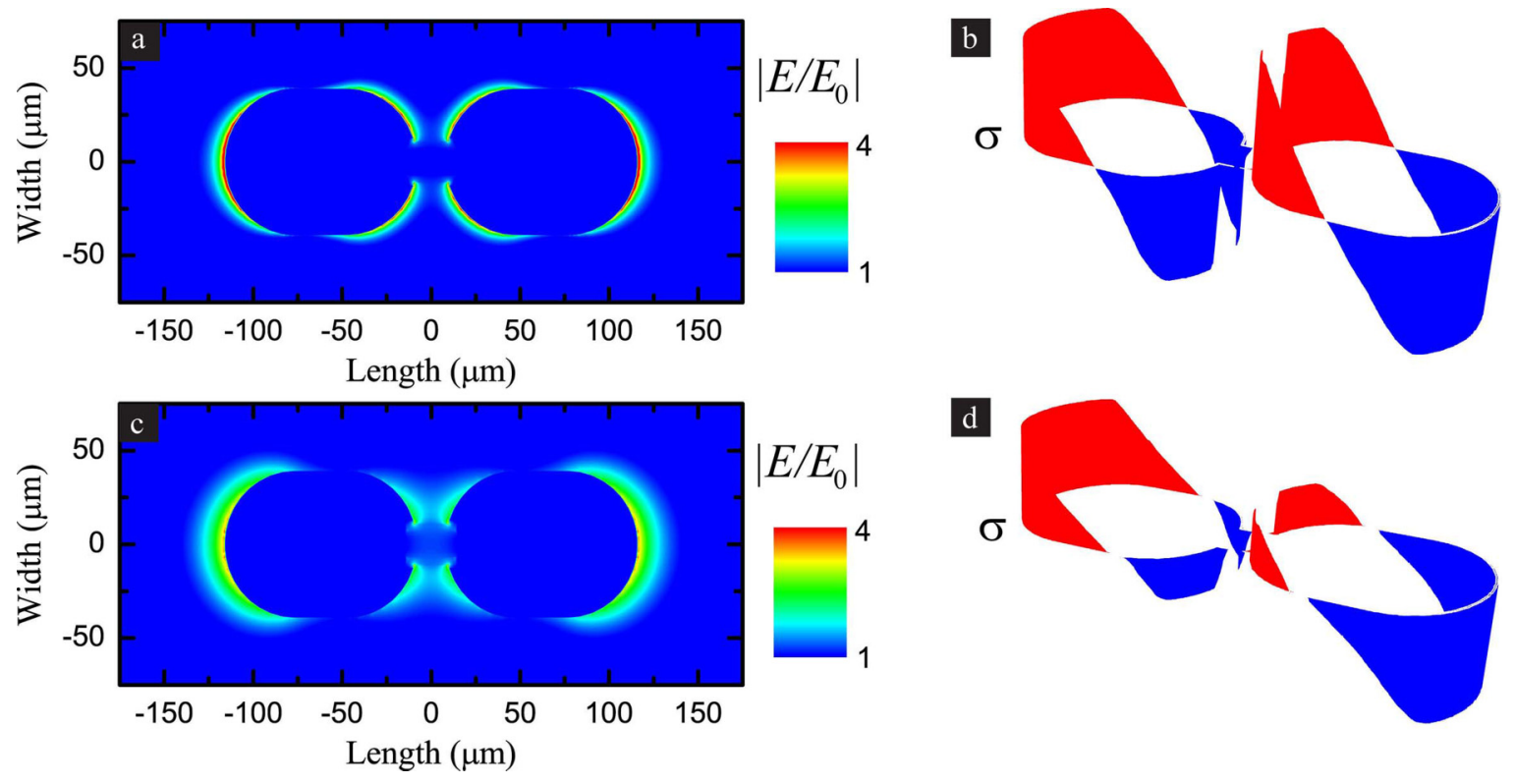

FIG. 3. (Color online) (a) Electric field enhancement $\left(\left|E / E_{0}\right|\right)$ and (b) surface charge distribution $(\sigma)$, calculated for an antenna with bridge width $8.6 \mu \mathrm{m}$, along an in-plane cross section of the antenna through its height center, at $\lambda=327 \mu \mathrm{m}$. (c) Electric field enhancement $\left(\left|E / E_{0}\right|\right)$ and (d) surface charge distribution of the same antenna at $\lambda=1030 \mu \mathrm{m}$. The red and blue areas in (b) and (d) denote positive and negative charges, respectively.

mode appears as a very broad, low-intensity mode. As the width of the bridge increases, charge transfer along the dimer is easier, and this mode becomes more intense and blue shifts, eventually becoming the antenna $\lambda / 2$ mode of a continuous rod, as can be seen in Fig. 2. For this mode the electric field (shown in Fig. 3(c) for $W_{\mathrm{b}}=8.6 \mu \mathrm{m}$ and at $\lambda=1030 \mu \mathrm{m}$ ) is distributed along the entire antenna, which resembles a long dipole, as observed also in the surface charge distribution of Fig. 3(d). This field distribution indicates an inductive behavior of the antenna load for this bridge width and wavelength. A non-negligible electric field intensity is also obtained around the bridge, associated with the presence of small surface charge densities of opposite sign at the crevices around the bridge.

An important parameter characterizing the resonant behavior of these loaded antennas is their impedance. The impedance determines the interaction of a dipole antenna with its load. More precisely, it is defined as the ratio of the input voltage over the displacement current and it quantifies the total amount of energy dissipated or stored in the system. In terms of the electromagnetic properties, the impedance is defined as,

$$
Z \equiv R-i X=\sqrt{\frac{\mu_{0} \mu}{\epsilon_{0} \epsilon}},
$$

where $R$ is the resistance, $X$ the reactance, $\mu_{0}=4 \pi \times$ $10^{-7}$ H.m ${ }^{-1}$ and $\epsilon_{0}=8.85 \times 10^{-12}$ F.m ${ }^{-1}$ are the vacuum permeability and permittivity respectively. The impedance is a complex quantity with its real component being twice the average power dissipated in the system and its imaginary component related to the difference between the average inductive and capacitive energies stored in the system [38].

The estimation of the complex impedance from the experimental data is difficult since it requires the complex transmitted and reflected signals [39]. However, in the simplest case, where there are no intrinsic magnetic fields due to circulating currents in the photogenerated antennas, we can assume that the magnetic permeability is equal to unity. Using this assumption the calculation of the impedance is simplified to only the knowledge of the complex transmitted signal.

In contrast to other works, where the optical properties and therefore the impedance of such loaded devices are estimated from the far-field transmission intensity measurements and assuming a lumped circuit model [27], here we measure the complex transmission and thus extract these values directly from the experimental data, i.e., without any a priori assumption. As it is demonstrated later in the text, the retrieved quantities are in a very good agreement with the far-field resonant frequencies. In the following we discuss the retrieval of the effective load impedance describing the random distribution of the antennas. This analysis is thus performed for the whole sample and not for single loaded antennas. In a conventional THz-TDS experiment the measured signal is the electric field as a function of time, which when Fourier transformed results in the complex electric field as a function of frequency, encompassing information about the amplitude and phase accumulated by the transmitted field. In order to retrieve the complex effective permittivity from transmission measurements we use the transfer matrix method [40]. In a sample that consists of a thin layer of material on a substrate (such as the one studied here), the wavelength is much longer than the thickness of the layer, $d \ll \lambda$. Therefore, the thin film approximation can be applied without any loss of generality. In this approximation the complex effective permittivity can be obtained from

$$
\frac{\tilde{E}_{\mathrm{sam}}(v)}{\tilde{E}_{\mathrm{sub}}(\nu)} \simeq \frac{1+i \beta \sqrt{\tilde{\epsilon}_{\mathrm{eff}}}}{1+i \beta \frac{\left(1-\sqrt{\tilde{\epsilon}_{\mathrm{eff}}}\right)\left(\sqrt{\tilde{\epsilon}_{\mathrm{eff}}}-\sqrt{\tilde{\epsilon}_{\mathrm{sub}}}\right)}{1+\sqrt{\tilde{\epsilon}_{\mathrm{sub}}}}},
$$



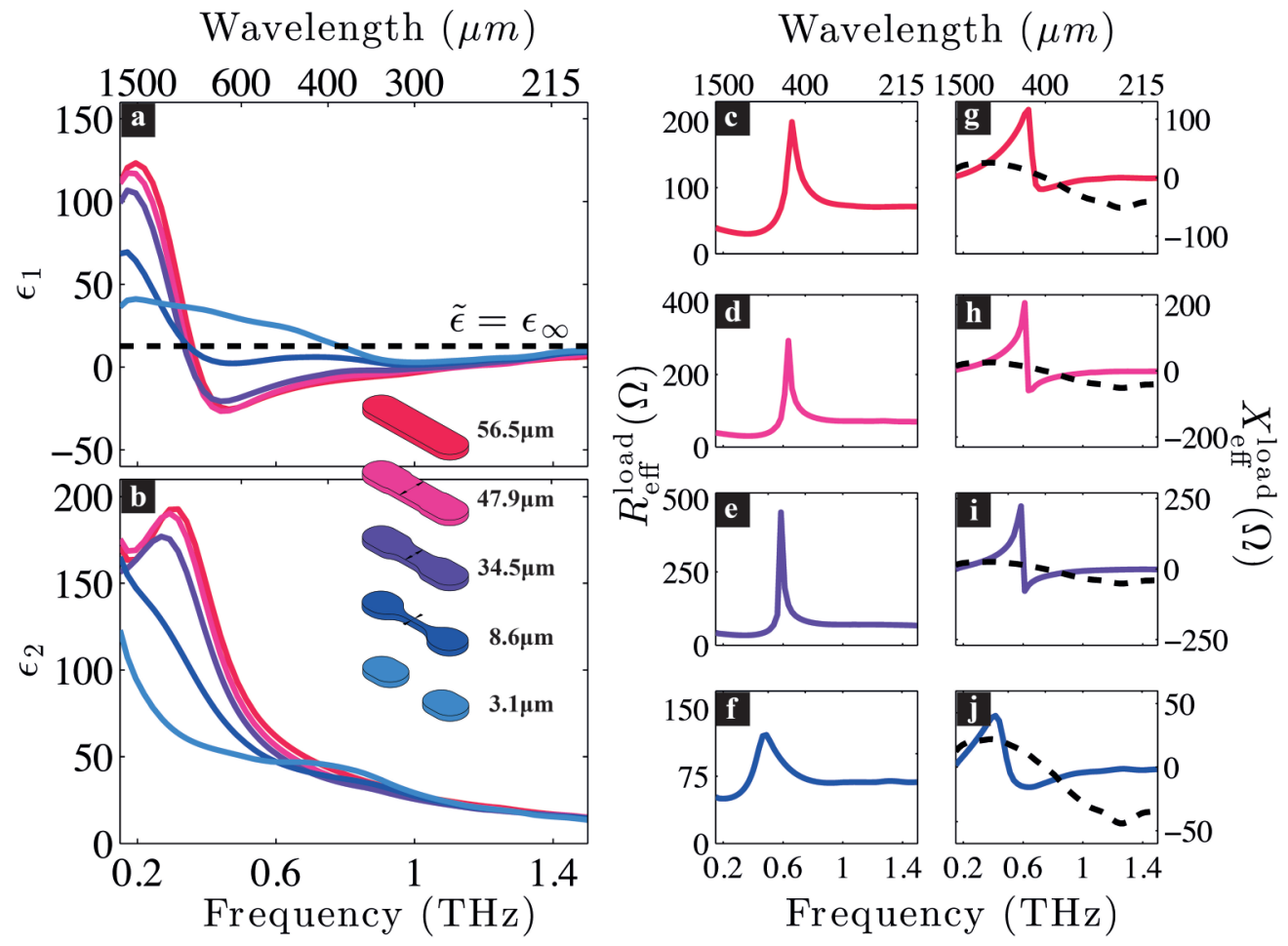

FIG. 4. (Color online) Detailed analysis of the transmitted signals through the photogenerated antennas. (a)-(b) Real ( $\left.\epsilon_{1}\right)$ and imaginary $\left(\epsilon_{2}\right)$ components of the effective permittivity for the randomly positioned loaded antennas as determined by the transfer matrix method in the thin film approximation. (c)-(f) Real component of the effective impedance (resistance, $\left.R_{\text {eff }}^{\text {load }}\right)$, (g)-(j) imaginary component of the effective impedance (reactance, $X_{\text {eff }}^{\text {load }}$ ). The black dashed lines illustrate the conjugate effective reactance of the randomly positioned dimer antennas $\left(X_{\text {eff }}^{* \text { dimer }}\right)$.

where $\tilde{E}_{\text {sam }}(\nu)$ and $\tilde{E}_{\text {sub }}(\nu)$ are the THz electric fields transmitted through the sample and through the substrate respectively, $\beta=\omega d / c, \omega$ is the angular frequency, $c$ is the speed of light in vacuum, $\tilde{\epsilon}_{\text {sub }}$ is the complex permittivity of the substrate and $\tilde{\epsilon}_{\text {eff }}$ is the effective complex permittivity of the photogenerated structures. The experimentally obtained effective permittivities for the loaded antennas are illustrated in Figs. 4(a)-4(b). The real component of the effective permittivity exhibits a resonant Drude-Lorentz-like behavior [41]. This response is characteristic for the motion of free charge carriers in the presence of an external driving force, which in our case is the THz electric field [42]. The frequency that corresponds to the transition through the $\tilde{\epsilon}_{\text {eff }}=\epsilon_{\infty}$ dashed line indicates the dominant resonant frequency of the antennas, where $\epsilon_{\infty}$ is the contribution of the ion lattice and bound charges to the permittivity of GaAs. This is also depicted in the imaginary component of the effective permittivity, which exhibits a resonant behavior. The increasing character of the imaginary component of the permittivity at low frequencies is due to the nonresonant absorption of $\mathrm{THz}$ radiation.

In order to define the effective permittivity of the load we divide the transmitted $\mathrm{THz}$ electric fields measured for the photogenerated loaded antennas [Figs. 1(a)-1(d)] with the transmission measured from the dimer antenna [Fig. 1(e)] [see Eq. (2) and Supplemental Material]. The obtained electric field corresponds to an effective response of the antenna load. This field is then normalized by the transmitted electric field obtained through a dark image as defined by the SLM. Consequently, the effective permittivity of the load is retrieved by using the transfer matrix method in the thin film approximation (see Supplemental Material). By defining the permittivity of the load we calculate the effective load impedance $\left(Z_{\text {eff }}^{\text {load }}\right)$ using Eq. (1). To calculate the impedance of the dimer, $Z_{\mathrm{eff}}^{\text {dimer }}$, we use an approach similar to the one described above, in which we normalize the $\mathrm{THz}$ electric field transmitted through the photogenerated dimers with the electric field taken when there are no pumped structures (reference). As the measurement that defines the impedance of the dimer contains also the contribution from the free charge carriers that are being generated in the background due to the finite contrast of the SLM, it is necessary to correct for this background. Therefore, we subtract the impedance of a dark image, $Z_{\text {eff }}^{\text {dark }}$.

Figures 4(c)-4(f) show the resistive $\left(R_{\text {eff }}\right)$ component while Figs. 4(g)-4(j) show the reactive $\left(X_{\text {eff }}\right)$ component of the effective impedance for different bridge widths, from $W_{\mathrm{b}}=$ $56.5 \mu \mathrm{m}$ (red) to $W_{\mathrm{b}}=8.6 \mu \mathrm{m}$ (blue). As can be observed in the figure, the real and imaginary components of the impedance show a resonant feature. An interesting aspect of the load behavior relies on the imaginary component of impedance [Figs. 4(g) -4(j)] that undergoes a change of sign from negative at high frequencies to positive at low frequencies. This change of sign is directly related to a transition from an inductive to a capacitive behavior of the effective load. It is also noteworthy that we observe a red shift of the resonance as we decrease the bridge size. This means that the transition from inductive to capacitive behavior also red shifts. From this analysis we observe experimentally that the behavior of the effective 


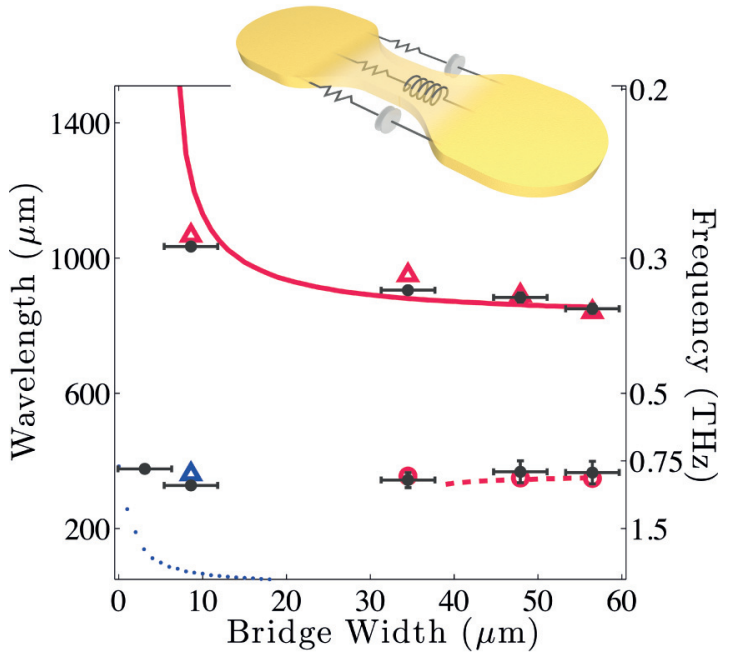

FIG. 5. (Color online) Lumped circuit model (lines) and experimental values (symbols) for varying bridge width. The blue dotted line corresponds to the capacitive mode for the $\lambda / 2$ mode of the individual component of the dimer, while the red solid and dashed lines correspond to the $\lambda / 2$ (long wavelengths) and $3 \lambda / 2$ (short wavelengths) inductive modes. The gray circles correspond to the resonant frequencies of the loaded antennas as obtained from a bi-Lorentzian fit to the far-field extinction measurements of Fig. 2. The blue/red triangles and red circles are the wavelength at which the effective dimer reactance equals to minus the effective load reactance of the measured sample as shown in Figs. $4(\mathrm{~g})-(\mathrm{j})\left(X_{\mathrm{eff}}^{\mathrm{dimer}}=-X_{\mathrm{eff}}^{\text {load }}\right)$. The inset illustrates a representation of the theoretical model that has been used for the calculations.

impedance exhibits a similar behavior to the impedance of a resonating $L C$ circuit connected in parallel, as it was previously predicted [24,43], where $L$ is the inductive load added by the conductive bridge and $C$ the parallel capacitive load introduced by the dielectric gap. In addition to this behavior, we show on the reactance plots the conjugate values of the reactance for the dimer, $X_{\text {eff }}^{*}$ dimer (dashed black line). The intersection points in the effective reactance plots [Figs. 4(g)-4(j)] correspond to the impedance matching conditions between the antenna and its load $\left(X_{\text {eff }}^{\text {dimer }}=-X_{\text {eff }}^{\text {load }}\right)$. The frequencies for which these impedance matching conditions are fulfilled are shown in Fig. 5 as circles and triangles, and they are in excellent agreement with the positions of the far-field resonances extracted from Fig. 2(a) and displayed as gray points in Fig. 5.

To qualitatively explain the spectral response of the loaded dimers we theoretically employ impedance matching conditions between the dimer antenna and its load. The impedance of a dimer antenna is similar to the response of an RF antenna [44] with the difference that its effective length is larger, due to its plasmonic nature and geometry [45]. The impedance of the load is defined by a parallel addition of the plasmonic bridge, $Z_{b}=i L_{\mathrm{g}} / \omega \epsilon_{\mathrm{b}} d W_{\mathrm{b}}$ and the dielectric gap, $Z_{\mathrm{g}}=i L_{\mathrm{g}} / \omega \epsilon_{\mathrm{g}} d\left(W-W_{\mathrm{b}}\right)$ [43], where $W$ and $W_{\mathrm{b}}$ are the experimentally obtained width of the gap and bridge respectively, $d$ is the thickness of the sample and $\epsilon_{\mathrm{b}, \mathrm{g}}$ are the permittivities of the bridge and gap calculated from the Drude model. For the calculation of the permittivities a carrier concentration of $10^{18} \mathrm{~cm}^{-3}$ for the bridge, $10^{16} \mathrm{~cm}^{-3}$ for the gap and $3 \times 10^{15} \mathrm{~cm}^{-3}$ for the surrounding semiconductor were used. An illustration of the model used for the calculation is shown in the inset of Fig. 5.

The results of this calculation display three distinct regimes as shown in Fig. 5. The first one, represented by the red solid line at long wavelengths, is the fundamental resonant mode, $\lambda / 2$, of the entire loaded antenna and corresponds to an inductive behavior. To confirm the inductive nature of the mode our FDTD simulations have shown that in contrast to the undoped GaAs layer, the magnetic field around the loaded antenna with bridge width $47.9 \mu \mathrm{m}$ has a fourfold enhancement and decreases as the bridge width becomes smaller. This mode diverges for small bridge widths due to the high resistance and capacitance added by the dielectric surrounding the bridge. Additionally, at short wavelengths we observe two modes. The first one for large bridge widths (red dashed line) corresponds to the higher-order resonant mode, $3 \lambda / 2$, of the entire resonant antenna while the second one for small bridge sizes (blue dotted line) is related to the $\lambda / 2$ resonant mode of the individual components of the dimer. The above theoretical calculations are in a very good agreement with the experimental data shown by gray points in Fig. 5.

\section{CONCLUSIONS}

In summary, we have demonstrated the all-optical generation of loaded plasmonic antennas for $\mathrm{THz}$ radiation on a flat GaAs layer using a spatial light modulator. The proposed concept allows a full control over the antenna properties and provides flexibility on defining the applied load. Furthermore, we are able to retrieve experimentally the effective impedance of the load and verify the validity of the lumped circuit theory in semiconducting systems by recovering a resonant behavior that is analogous to an $L C$ circuit. We have also shown the existence of a capacitive and an inductive behavior of localized surface plasmon polaritons at $\mathrm{THz}$ frequencies. Our results indicate that the transition between these two regimes can be actively controlled by tuning the design of the loaded antennas, which is controlled by the optical illumination pattern on a flat GaAs layer. The demonstration of active semiconductor lumped circuits and the possibility of optically generating these circuits will open new venues for fundamental research and new technologies. Examples of these are the ultrafast dynamics of photogenerated circuits [46] or their applications for resonant $\mathrm{THz}$ sensing and spectroscopy $[47,48]$ on flat layers that are optically structured.

\section{ACKNOWLEDGMENTS}

We are thankful to H. K. Tyagi and J. Versluis for valuable discussions. The authors would also like to acknowledge P. Mulder, G. J. Bauhuis, and J. J. Schermer for the sample fabrication. This work has been supported by the ERC through Grant No. 259727 THZ-PLASMON and by the Netherlands Foundation for Fundamental Research on Matter (FOM) and the Netherlands Organization for Scientific Research (NWO). J.A. and C.T. acknowledge support from Project FIS201341184-P from the Spanish MINECO and Project IT756-13 of the Department of Education of the Basque Government. 
[1] P. Bharadwaj, B. Deutsch, and L. Novotny, Adv. Opt. Photon. 1, 438 (2009).

[2] P. Mühlschlegel, H.-J. Eisler, O. Martin, B. Hecht, and D. Pohl, Science 308, 1607 (2005).

[3] P. Biagioni, J.-S. Huang, and B. Hecht, Rep. Prog. Phys. 75, 024402 (2012).

[4] F. Neubrech, A. Pucci, T. W. Cornelius, S. Karim, A. GarcíaEtxarri, and J. Aizpurua, Phys. Rev. Lett. 101, 157403 (2008).

[5] M. W. Knight, H. Sobhani, P. Nordlander, and N. J. Halas, Science 332, 702 (2011).

[6] N. Yu, P. Genevet, M. A. Kats, F. Aieta, J.-P. Tetienne, F. Capasso, and Z. Gaburro, Science 334, 333 (2011).

[7] P. Ghenuche, S. Cherukulappurath, T. H. Taminiau, N. F. van Hulst, and R. Quidant, Phys. Rev. Lett. 101, 116805 (2008).

[8] A. F. Koenderink, Nano Letters 9, 4228 (2009).

[9] A. G. Curto, G. Volpe, T. H. Taminiau, M. P. Kreuzer, R. Quidant, and N. F. van Hulst, Science 329, 930 (2010).

[10] F. González and G. Boreman, Infrared Phys. Tech. 46, 418 (2005).

[11] H. Fischer and O. J. F. Martin, Opt. Express 16, 9144 (2008).

[12] H. Guo, T. P. Meyrath, T. Zentgraf, N. Liu, L. Fu, H. Schweizer, and H. Giessen, Opt. Express 16, 7756 (2008).

[13] A. M. Funston, C. Novo, T. J. Davis, and P. Mulvaney, Nano Letters 9, 1651 (2009).

[14] B. Luk'yanchuk, N. I. Zheludev, S. A. Maier, N. J. Halas, P. Nordlander, H. Giessen, and C. T. Chong, Nature Mater. 9, 707 (2010).

[15] A. E. Miroshnichenko, S. Flach, and Y. S. Kivshar, Rev. Mod. Phys. 82, 2257 (2010).

[16] Y. Francescato, V. Giannini, and S. A. Maier, ACS Nano 6, 1830 (2012).

[17] M. Abb, Y. Wang, P. Albella, C. H. de Groot, J. Aizpurua, and O. L. Muskens, ACS Nano 6, 6462 (2012).

[18] A. Lovera, B. Gallinet, P. Nordlander, and O. J. F. Martin, ACS Nano 7, 4527 (2013).

[19] N. Papasimakis, V. A. Fedotov, N. I. Zheludev, and S. L. Prosvirnin, Phys. Rev. Lett. 101, 253903 (2008).

[20] N. Liu, L. Langguth, T. Weiss, J. Kästel, M. Fleischhauer, T. Pfau, and H. Giessen, Nature Mater. 8, 758 (2009).

[21] S. Sun, Q. He, S. Xiao, Q. Xu, X. Li, and L. Zhou, Nature Mater. 11, 426 (2012).

[22] P. Genevet, N. Yu, F. Aieta, J. Lin, M. A. Kats, R. Blanchard, M. O. Scully, Z. Gaburro, and F. Capasso, Appl. Phys. Lett. 100, 013101 (2012).

[23] N. Engheta, A. Salandrino, and A. Alù, Phys. Rev. Lett. 95, 095504 (2005).

[24] A. Alù and N. Engheta, Phys. Rev. Lett. 101, 043901 (2008).

[25] N. Liu, F. Wen, Y. Zhao, Y. Wang, P. Nordlander, N. J. Halas, and A. Alú, Nano Letters 13, 142 (2013).
[26] M. Schnell, A. Garcia-Etxarri, A. Huber, K. Crozier, J. Aizpurua, and R. Hillenbrand, Nature Photon. 3, 287 (2009).

[27] Y. Wang, M. Abb, S. A. Boden, J. Aizpurua, C. H. de Groot, and O. L. Muskens, Nano Letters 13, 5647 (2013).

[28] M. Abb, P. Albella, J. Aizpurua, and O. L. Muskens, Nano Letters 11, 2457 (2011).

[29] J. Gómez Rivas, M. Kuttge, P. H. Bolivar, H. Kurz, and J. A. Sánchez-Gil, Phys. Rev. Lett. 93, 256804 (2004).

[30] A. J. Huber, F. Keilmann, J. Wittborn, J. Aizpurua, and R. Hillenbrand, Nano Letters 8, 3766 (2008).

[31] H.-T. Chen, W. J. Padilla, J. M. O. Zide, A. C. Gossard, A. J. Taylor, and R. D. Averitt, Nature (London) 444, 597 (2006).

[32] E. A. Shaner, J. G. Cederberg, and D. Wasserman, Appl. Phys. Lett. 91, 181110 (2007).

[33] H.-T. Chen, H. Lu, A. K. Azad, R. D. Averitt, A. C. Gossard, S. A. Trugman, J. F. O'Hara, and A. J. Taylor, Opt. Express 16, 7641 (2008).

[34] J. Gu, R. Singh, X. Liu, X. Zhang, Y. Ma, S. Zhang, S. A. Maier, Z. Tian, A. K. Azad, H.-T. Chen, A. J. Taylor, J. Han, and W. Zhang, Nature Commun. 3, 1151 (2012).

[35] G. Georgiou, H. K. Tyagi, P. Mulder, G. J. Bauhuis, J. J. Schermer, and J. Gómez Rivas, Sci. Rep. 4, 3584 (2014).

[36] See Supplemental Material at http://link.aps.org/supplemental/ 10.1103/PhysRevB.91.125443 for more information on the CCD camera images, carrier dynamics and the retrieval of the impedance from the experimental data.

[37] O. Pérez-González, N. Zabala, A. G. Borisov, N. J. Halas, P. Nordlander, and J. Aizpurua, Nano Letters 10, 3090 (2010).

[38] R. B. Adler, L. J. Chu, and R. M. Fano, Electromagnetic Energy Transmission and Radiation (Wiley, New York, 1960).

[39] D. R. Smith, D. C. Vier, T. Koschny, and C. M. Soukoulis, Phys. Rev. E 71, 036617 (2005).

[40] Y. Pochi, Optical Waves in Layered Media (Wiley, New York, 2005).

[41] R. Ulbricht, E. Hendry, J. Shan, T. F. Heinz, and M. Bonn, Rev. Mod. Phys. 83, 543 (2011).

[42] A. Agrawal, Z. V. Vardeny, and A. Nahata, Opt. Express 16, 9601 (2008).

[43] A. Alù and N. Engheta, Nature Photon. 2, 307 (2008).

[44] C. A. Balanis, Antenna theory: Analysis and Design (Wiley, New York, 2012).

[45] L. Novotny, Phys. Rev. Lett. 98, 266802 (2007).

[46] H. K. Tyagi and J. Gómez-Rivas, J. Opt. (Bristol, U.K.) 16, 094011 (2014).

[47] A. Berrier, M. C. Schaafsma, G. Nonglaton, J. Bergquist, and J. Gómez-Rivas, Biomed. Opt. Express 3, 2937 (2012).

[48] S. J. Park, J. T. Hong, S. J. Choi, H. S. Kim, W. K. Park, S. T. Han, J. Y. Park, S. Lee, D. S. Kim, and Y. H. Ahn, Sci. Rep. 4, 4988 (2014). 